\title{
Size distributions of Suspended Fine Particles during the Cleaning of an Office
}

\author{
Jun-Ho Ji* \\ EcoPictures Co. Ltd., Seoul 05855, Korea
}

\begin{abstract}
In this study, the concentration and size distribution of fine dust particles were analyzed by measuring the dust in air generated during the cleaning of an indoor office. We measured the $\mathrm{PM}_{10}, \mathrm{PM}_{2.5}$, and $\mathrm{PM}_{1.0}$ and analyzed the size distributions of particles larger than $0.3 \mu \mathrm{m}$ in diameter during cleaning. The results showed that the concentration of $\mathrm{PM}_{10}$ increased rapidly during cleaning, whereas the $\mathrm{PM}_{1.0}$ did not increase. Prior to sweeping the floor with a broom, the fine dust concentration was $\sim 50 \mu \mathrm{g} \mathrm{m}^{-3}$, but it increased to $\sim 400 \mu \mathrm{g} \mathrm{m}^{-3}$ as the sweeping progressed. When a vacuum cleaner was used, the concentration of $\mathrm{PM}_{10}$ increased, but the increase in $\mathrm{PM}_{2.5}$ was relatively small. As in the case of sweeping, the $\mathrm{PM}_{1.0}$ did not increase while vacuuming.
\end{abstract}

Keywords: Indoor cleaning; Broom; Vacuum cleaner; $\mathrm{PM}_{10} ; \mathrm{PM}_{2.5} ; \mathrm{PM}_{1.0}$.

\section{INTRODUCTION}

There are various reports that fine dust and ultrafine dust are severe health hazards. Recently, there is a great demand for air quality improvements worldwide. The annual average concentration of $\mathrm{PM}_{10}$ in Seoul was $44 \mu \mathrm{g} \mathrm{m} \mathrm{m}^{-3}$ in 2013, $46 \mu \mathrm{g} \mathrm{m}^{-3}$ in $2014,45 \mu \mathrm{g} \mathrm{m}^{-3}$ in 2015 , and $48 \mu \mathrm{g} \mathrm{m}^{-3}$ in 2016 although it had decreased from $61 \mu \mathrm{g} \mathrm{m}^{-3}$ in 2007 to $41 \mu \mathrm{g} \mathrm{m}^{-3}$ in 2012. However, it is still higher than the developed countries and especially higher than twice the $20 \mu \mathrm{g} \mathrm{m}^{-3}$ that is the annual mean $\mathrm{PM}_{10}$ concentration of the World Health Organization (WHO) air quality guideline. It is difficult to reduce the human exposure to airborne particles only by control of domestic air pollution. Therefore, the short-term solution is to reduce the suspended particles indoors where people spend most of their time.

According to a recent study, Korean residents spend more than 21 hours a day indoors, and their residence time in a space differed depending on occupation, sex, and age (Yoon et al., 2017). However, there are a few data analyzed in detail on the exposure to suspended dusts according to the behavior patterns of various people in the indoor environment and on

\footnotetext{
* Corresponding author.

Tel.: +82-2-6959-5899; Fax: +82-2-6959-5898

E-mail address: caputjun@gmail.com
}

This article is an English version of "Size distributions of suspended fine particles during cleaning in an office [Korean]" published in Particle and Aerosol Research in June 2018. the characteristics of the sources (Bae and Kim, 2017). As a result of exposure studies for suspended dust indoor, it has been reported that aerosols can be released from vacuum cleaners during indoor cleaning (Ozturk, 2006; Knibbs et al., 2012; Wu et al., 2012; Lee et al., 2014). Wu et al. (2012) analyzed the amount of dust generated from a vacuum cleaner in a small chamber by measuring the number density and size distribution based on it. Knibbs et al. (2012) confirmed the emission characteristics of dust generated by conducting duct tests of 21 kinds of vacuum cleaners. Lee et al. (2014) confirmed that fine dust could be released during the operation of the vacuum cleaner through the test, which drove a vacuum cleaner in the chamber. Nevertheless, there is a lack of research on concentration properties and size distribution of dust generated in actual cleaning process.

In this study, we investigated the characteristics of dust generated in the process of cleaning in an office where people wear shoes. $\mathrm{PM}_{10}, \mathrm{PM}_{2.5}$, and $\mathrm{PM}_{1.0}$ were analyzed, and the particle size distributions of suspended dust larger than $0.25 \mu \mathrm{m}$ was measured and analyzed in the cleaning process. In particular, the characteristics of dust that resuspended in the process of using broom or vacuum cleaner were analyzed.

\section{METHODS}

The measurements were carried out at an office having an area of $105.3 \mathrm{~m}^{2}$ located on the $13^{\text {th }}$ floor of the newly constructed building in Seoul. The office zones were divided only by partitions of about $1.5 \mathrm{~m}$ in height. As a first case, we measured the particles generated in the process of cleaning the office floor with a broom. There was a lot of dust on the floor because it was office moving day. At the second and third measurements, a vacuum cleaner was used 
for floor cleaning. Dust suctioned by the vacuum cleaner is collected in a large dust bag installed in the vacuum cleaner, and the filtered air is discharged into air by passing through a filter equipped at the air outlet. Secondly, in three weeks after the moving, the characteristics of the fine particles in air were measured and analyzed during the cleaning process using the vacuum cleaner. It was a cloudy day with a rainfall of about $6 \mathrm{~mm}$. In four weeks from the moving day, the third measurement was performed. An optical particle counter (OPC; Model 11A; GRIMM, Germany) was used in all three measurements. A condensation particle counter (CPC; Model 5400; GRIMM, Germany) was additionally used at the third measurement. The number concentration of particles larger than $4 \mathrm{~nm}$ was measured. The interior structure including arrangement of desk, table, chair, and so on of the relocated office and the cleaning circulation line are shown in Fig. 1. Measurement instruments were located at the starting point of cleaning. The conditions for three cleaning cases using a broom and a vacuum cleaner in the office are summarized in Table 1.

\section{RESULTS AND DISCUSSION}

\section{Concentration and Size Distribution of Dust Suspended in Cleaning with a Broom}

The first measurement was made on the day we moved to the office. Since there was a lot of dust on the floor, we used a broom for sweeping the floor dust. The suspended particles in air in this cleaning process was measured. During the cleaning process, we opened all the windows. There was little wind outside though the office entrance door was opened to the corridor. As shown in Fig. 1, the cleaning with a broom started from where the measuring instrument was located.

Fig. 2 shows the changes in the concentration of particles suspended in the cleaning process and after cleaning. Fig. 2(a) shows the changes of $\mathrm{PM}_{10}, \mathrm{PM}_{2.5}$, and $\mathrm{PM}_{1.0}$ before, during, and after cleaning. The $\mathrm{PM}_{10}$ concentration was about $50 \mu \mathrm{g} \mathrm{m}^{-3}$ before cleaning, but it increased to about $400 \mu \mathrm{g} \mathrm{m}^{-3}$ during cleaning with broom. Cleaning with a broom was performed for about 15 minutes. When cleaning around the measuring device, the concentration increased the most. While moving away from the measuring site, the indoor dust concentration decreased first and then increased. Total amounts of indoor suspended dust increased with sweeping using a broom. Dust concentration in air was increased with time because the suspended fine dust diffuses in the total office space. It took at least 1 hour for the indoor concentration decreasing to about $50 \mu \mathrm{g} \mathrm{m}^{-3}$ even after cleaning. The air exchange by natural ventilation was little due to the structure of the office although the windows were opened during and after the cleaning. In Fig. 2(b), the mass concentrations of $\mathrm{PM}_{2.5-1.0}, \mathrm{PM}_{10-2.5}$, the relatively large dust of which particle size is in a region of 1.0-2.5 and 2.5-10 $\mu \mathrm{m}$, and $\mathrm{PM}_{1.0}$ were indicated which were calculated from $\mathrm{PM}_{10}, \mathrm{PM}_{2.5}$, and $\mathrm{PM}_{1.0}$ data. The concentration of $\mathrm{PM}_{10-2.5}$ increased sharply while that of $\mathrm{PM}_{1.0}$ did not change much. The reason might be that the dust on the floor is resuspended in the air due to the physical force such as sweeping with broom, but the dust smaller than $1 \mu \mathrm{m}$ seems not falling well from the large dust. Fig. 2(c) shows the ratio of concentration after cleaning to before cleaning. For the before-cleaning concentration,

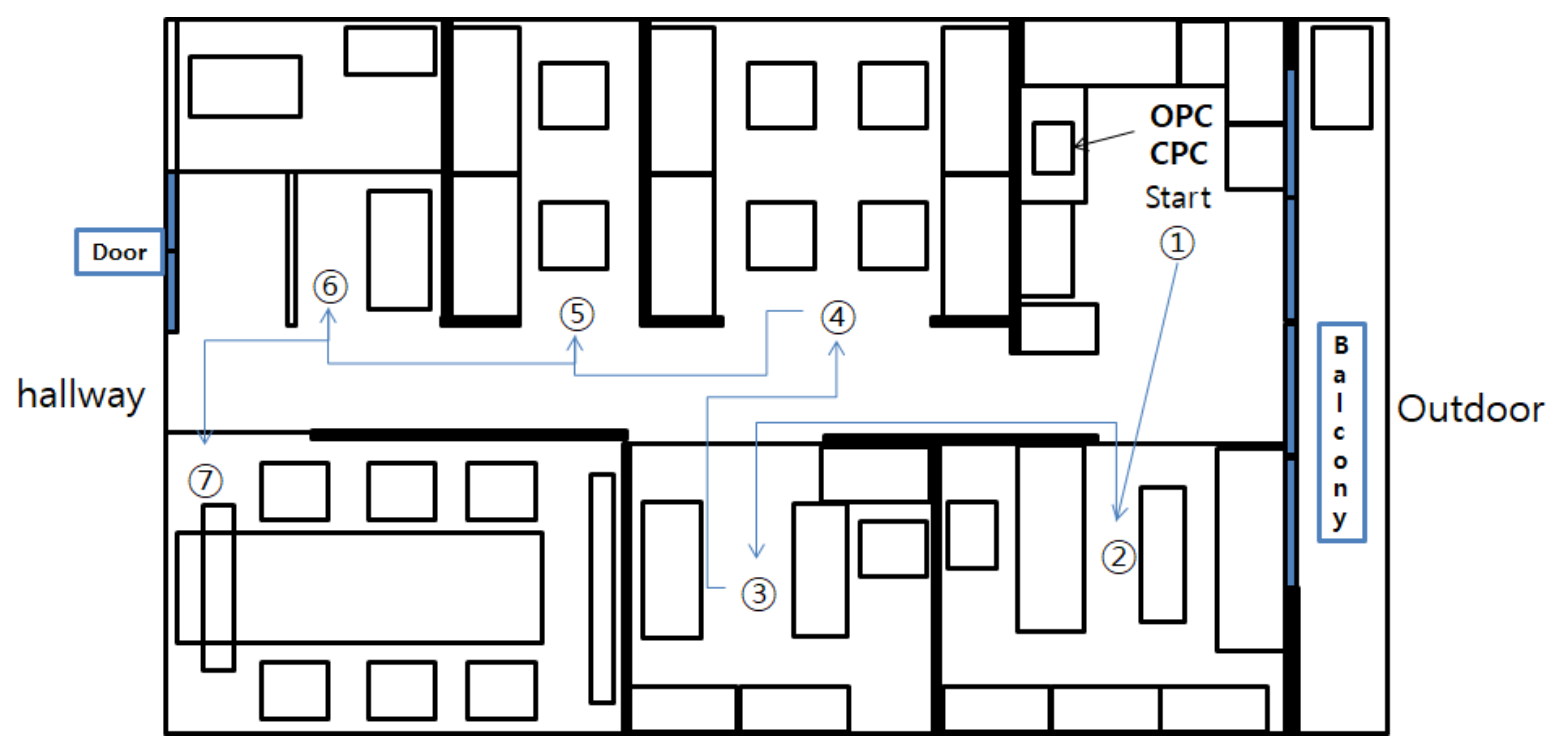

Fig. 1. Office indoor structure and cleaning moving line.

Table 1. Indoor cleaning conditions and cleaning tools.

\begin{tabular}{llll}
\hline Case & Cleaning method & Cleaning activity & Amount of dust on the floor \\
\hline 1 & Broom & Office cleaning after moving & Large \\
2 & Vacuum cleaner & Vacuum cleaning & Small (rainy day) \\
3 & Vacuum cleaner & Vacuum cleaning & Small \\
\hline
\end{tabular}


(a)

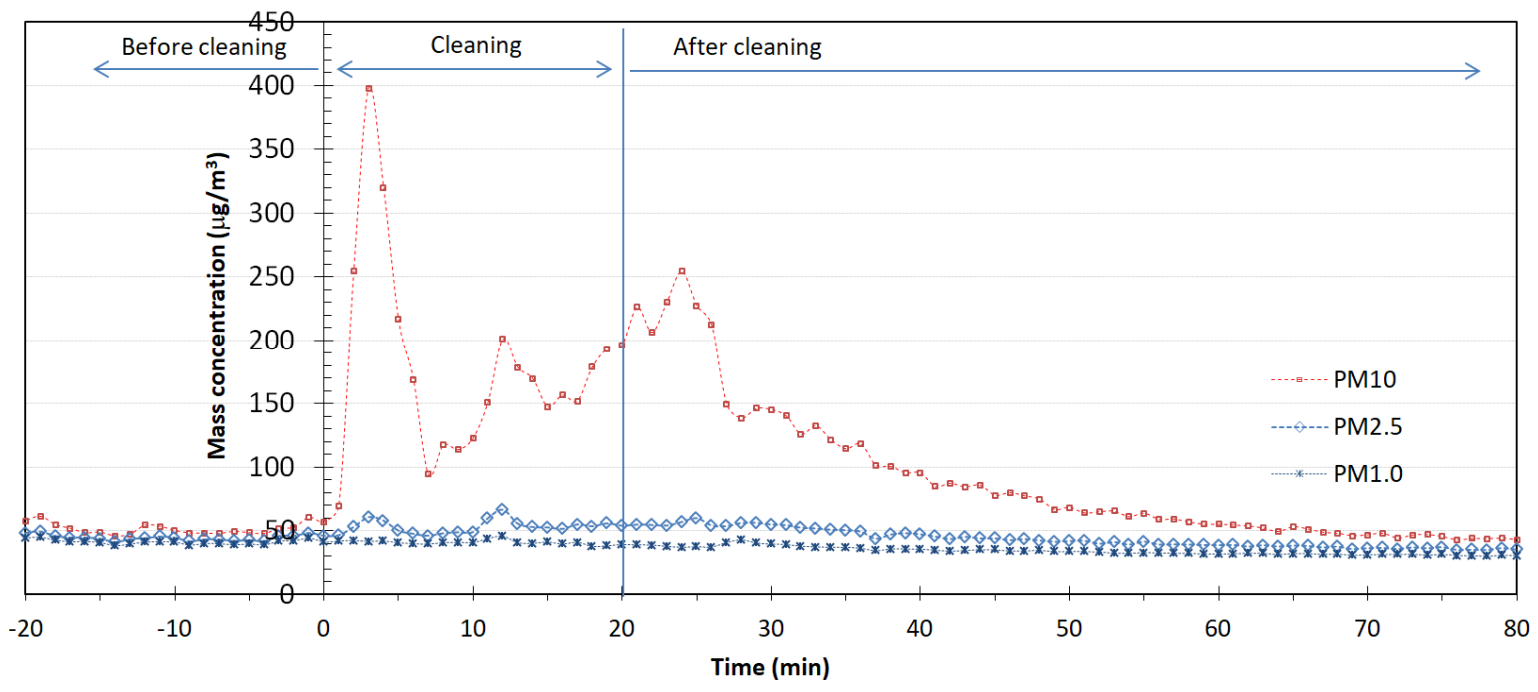

(b)

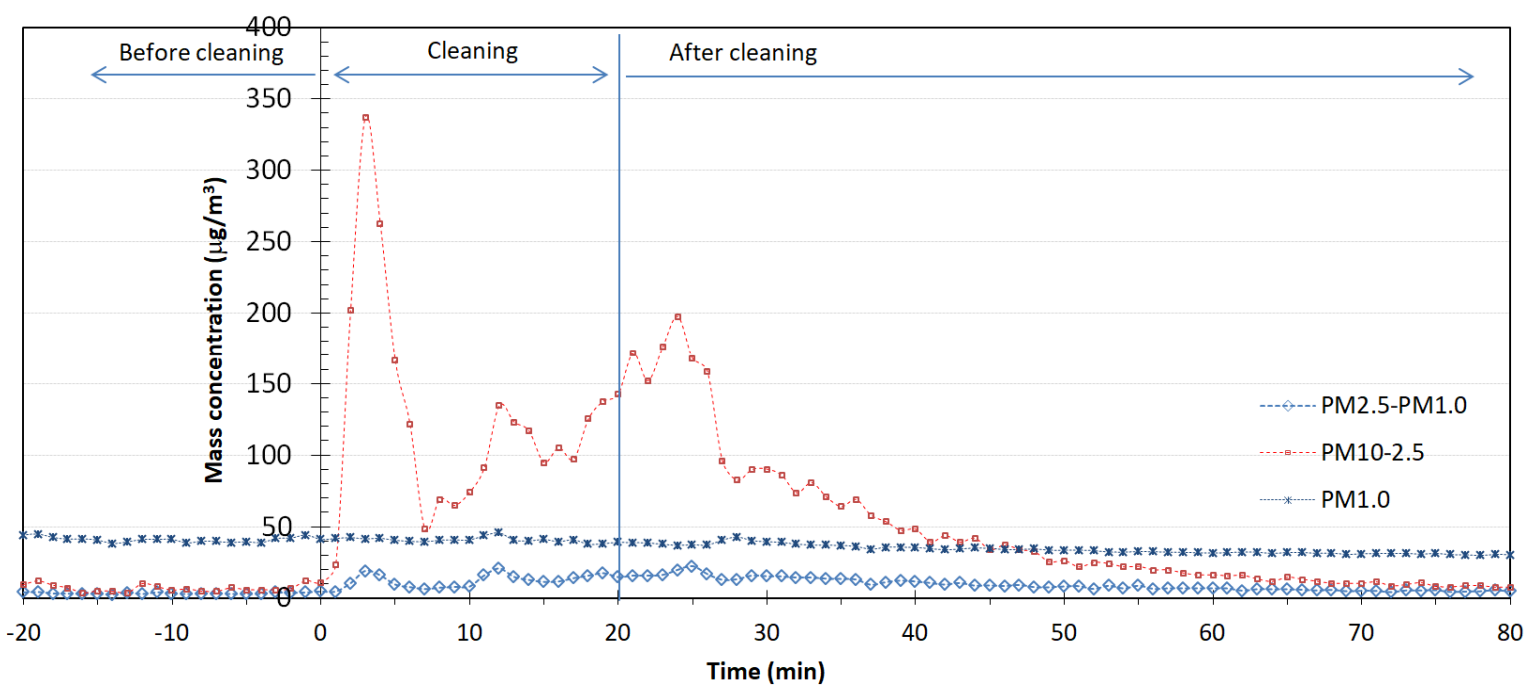

(c)

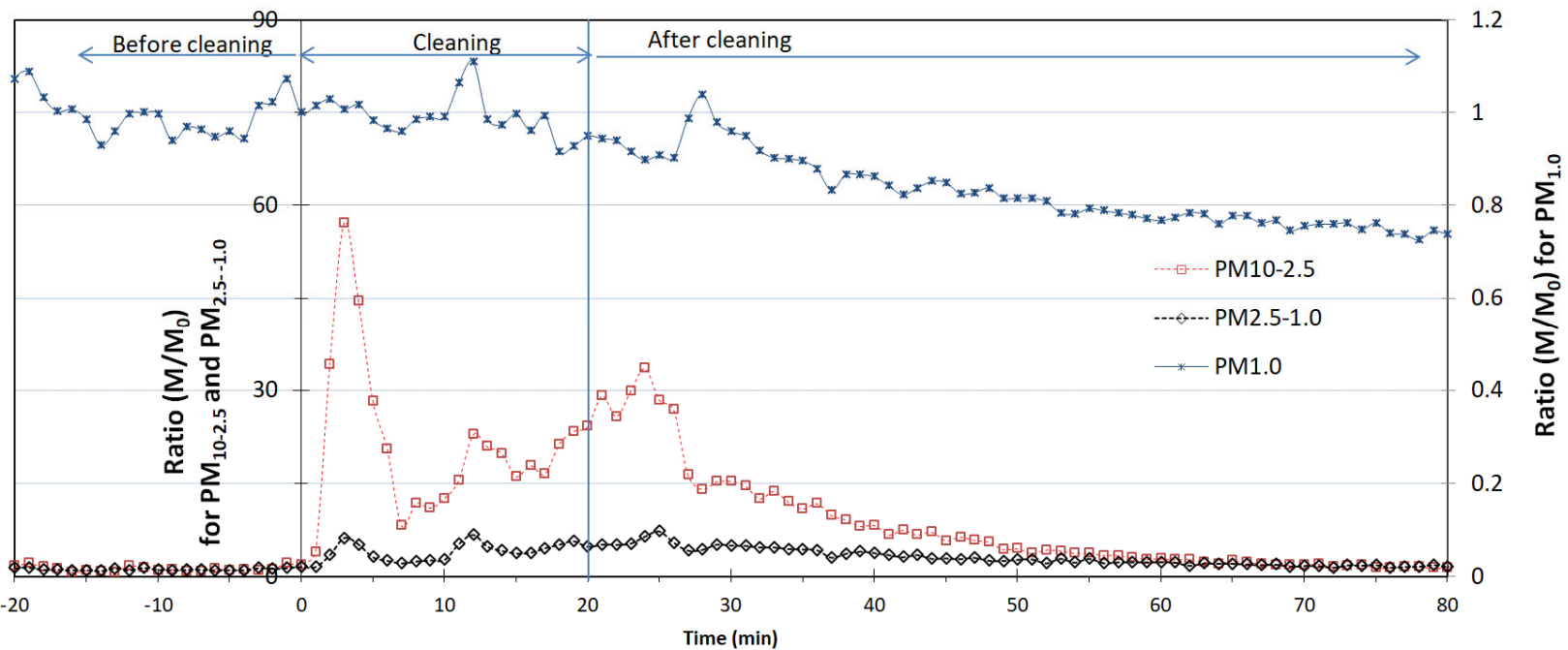

Fig. 2. Mass concentrations and ratios of mass concentrations of particles resuspended during cleaning with a broom.

10 minute average value was used. The particle having the size of $1-2.5 \mu \mathrm{m}$ increased about 5 times whereas the relatively large dust having a diameter of $2.5-10 \mu \mathrm{m}$ increased about 60 times than before cleaning. The particles smaller than $1 \mu \mathrm{m}$ did not show a clear increase trend.

Fig. 3 shows the size distribution change of the dust 
suspended in the cleaning process using a broom. The basic data of the OPC shows the number concentration of each size channel, but the measured size was expressed as an optically equivalent size in this study to qualitatively compare the change characteristics of a dust according to its relative size. Each dust was assumed to have a spherical shape and a density of $1.0 \mathrm{~g} \mathrm{~cm}^{-3}$, and the particle size distribution was estimated. In order to express the optical equivalent size as an aerodynamic size, it is necessary to know the exact density. Since the particle density is assumed to be $1.0 \mathrm{~g} \mathrm{~cm}^{-3}$, the particle size distribution must be shifted to the smaller side (Hinds, 1999). The particle size distribution before cleaning was like that of particles in the atmosphere. Two peaks were found in the size distribution that one smaller than $1 \mu \mathrm{m}$, and the other larger than $1 \mu \mathrm{m}$.

At the beginning of the cleaning, there is almost no change in the concentration of dust having size below $1 \mu \mathrm{m}$ as shown in Fig. 2, but the concentration of particles in the range of $1-10 \mu \mathrm{m}$ increases sharply. It indicates that the $1-$ $10 \mu \mathrm{m}$ particles were resuspended into air from the floor by sweeping with a broom like that the dust is generated by the wind. Because there was a lot of dust on the floor during the moving process, the dust was resuspended during the cleaning process and the concentration of particles suspended in air was greatly increased. It was confirmed that the particles larger than $10 \mu \mathrm{m}$, which are difficult to track merely from $\mathrm{PM}_{10}, \mathrm{PM}_{2.5}$, and $\mathrm{PM}_{1.0}$ data of Fig. 2, were suspended in the air by particle size distribution analysis.

\section{Concentration and Size Distribution of Dust Suspended in Cleaning with a Vacuum Cleaner}

Fig. 4 shows the results of measuring the dust suspended during the vacuum cleaning. It was in three or four weeks after moving, there was not much dust on the floor due to

(a)

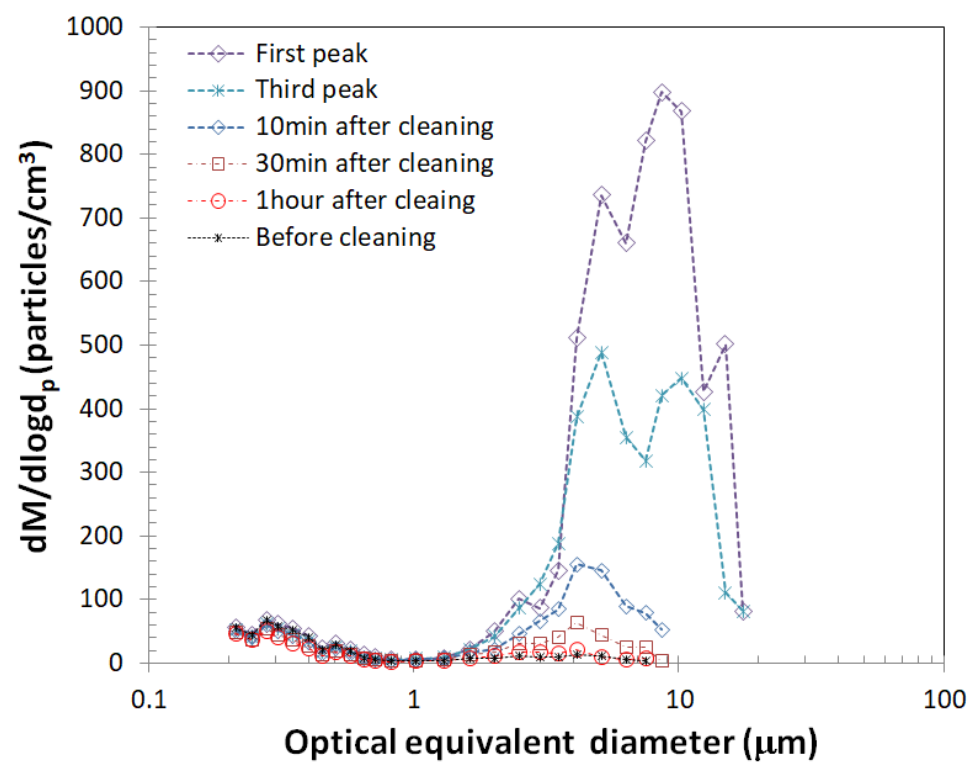

(b)

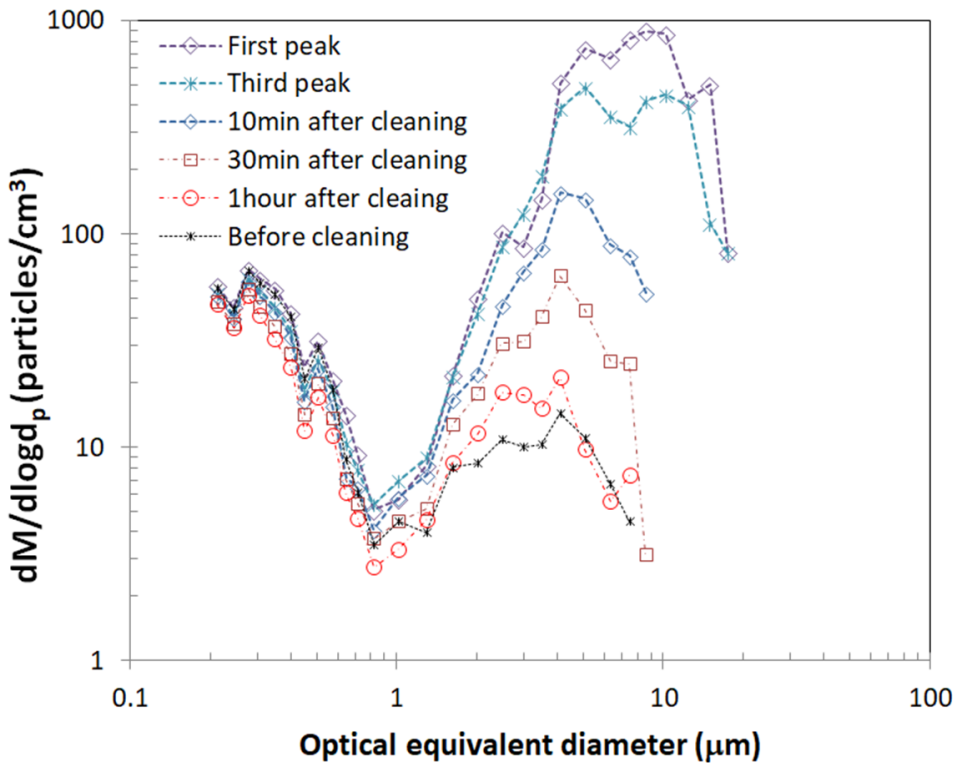

Fig. 3. Particle size distributions of resuspended dust during cleaning with a broom. 
(a)

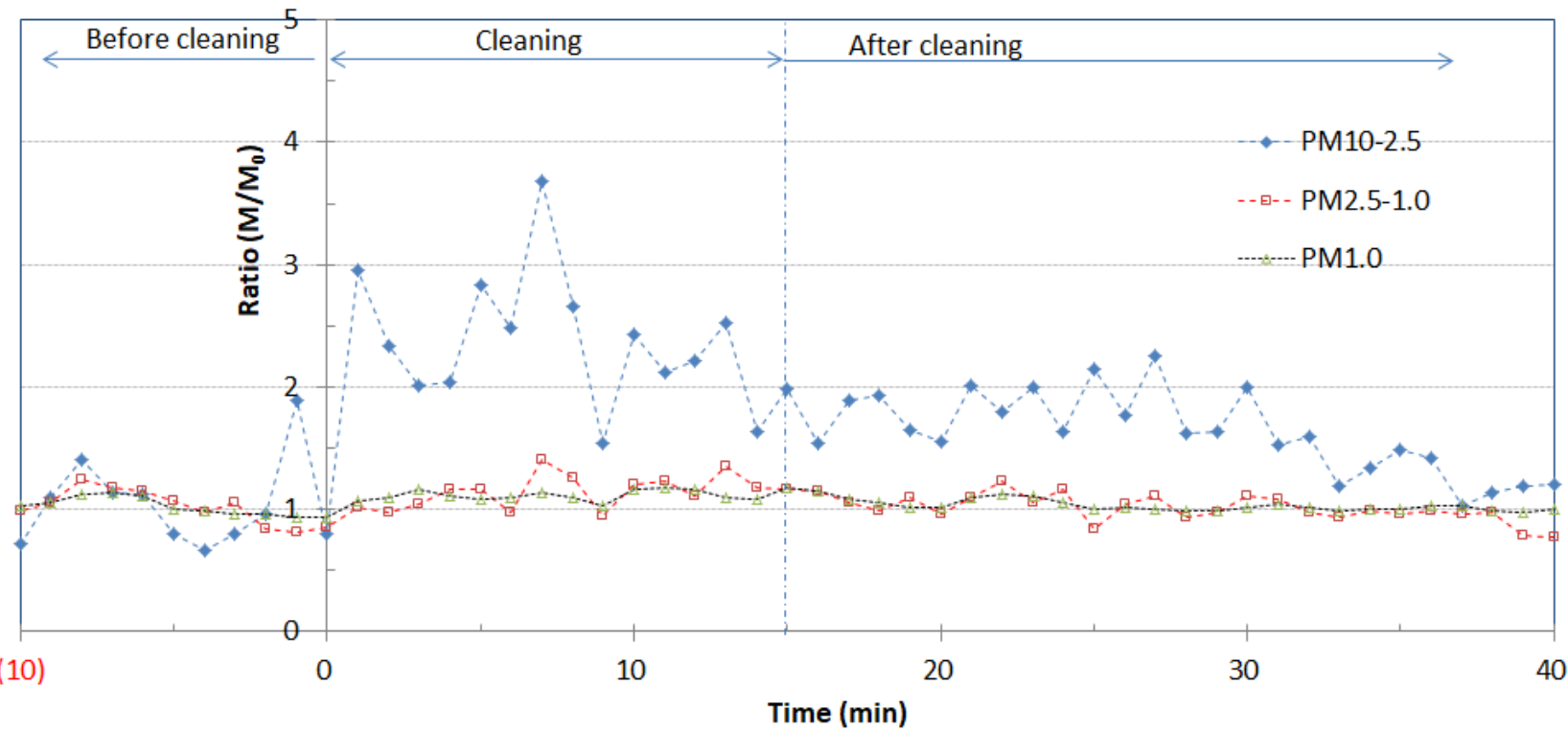

(b)

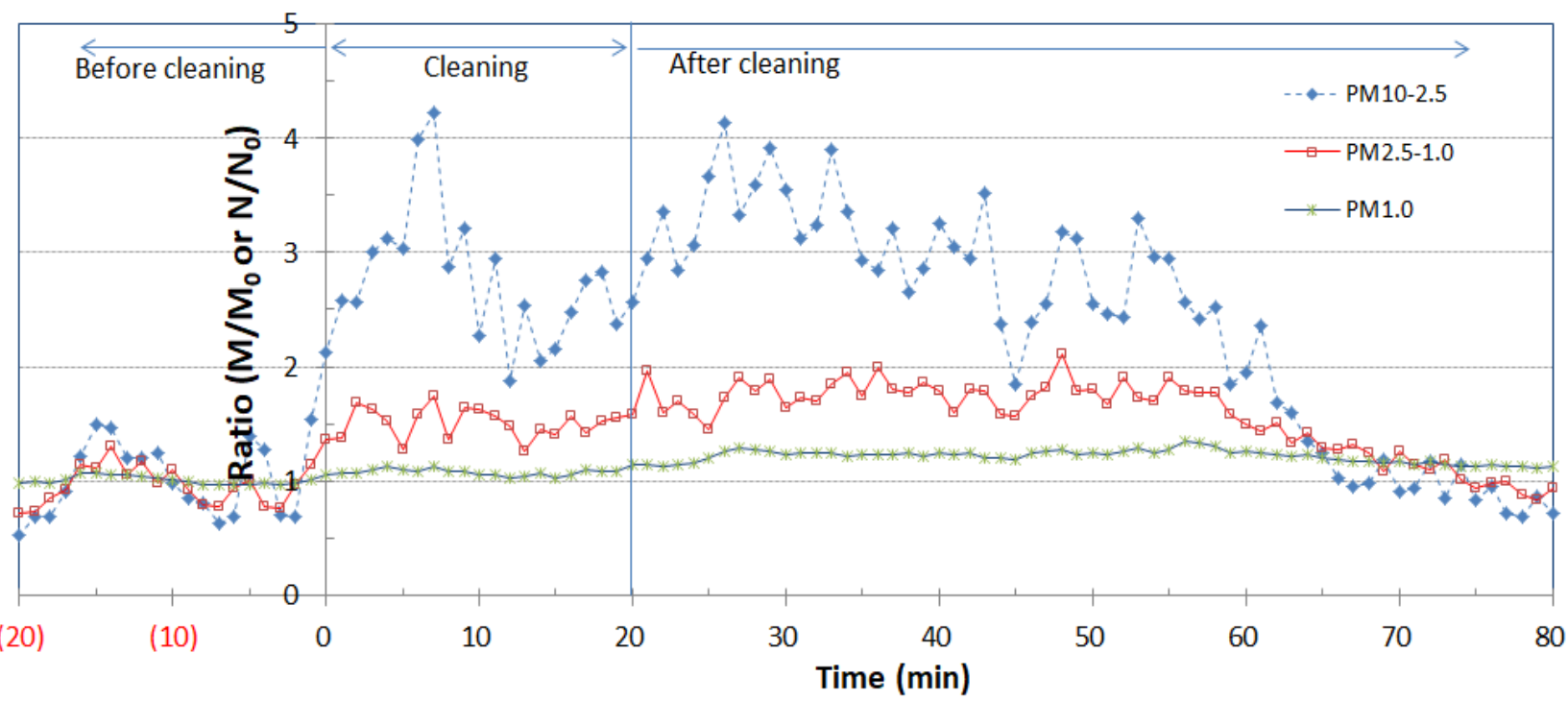

Fig. 4. Mass concentrations of particles resuspended during cleaning with a vacuum cleaner on a (a) rainy day and (b) sunny day.

steadily cleaning and mopping. It was a condition that only a certain amount of dust was on the floor due to dust shaken out of shoes. Fig. 4(a) shows the result of cloudy days with rainfall of about $6 \mathrm{~mm}$ at three weeks after moving. On the other hand, Fig. 4(b) shows the result of clear and dry day after four weeks after moving. The increase in indoor dust concentration was lower when using vacuum cleaner than when the floor was cleaned with a broom in the dusty condition on the moving day shown in Fig. 2. Compared with before cleaning, the mass concentration of the dust in the range of $2.5-10 \mu \mathrm{m}$ was less than 5 times when using a vacuum cleaner with little dust on the floor. It was unlike the case where it increased by 60 times when using a broom for cleaning. The cause of such a result is the difference between a broom and a vacuum cleaner, and a difference in the amount of floor dust. Particularly, in Fig. 3, there was almost no resuspension of particles having size less than $1 \mu \mathrm{m}$ as well as in the range of $1-2.5 \mu \mathrm{m}$. Those particles were hard to fall off from relatively large dusts because it was a wet weather day with rainfall. On a dry and sunny day, the increased concentration of 1-2.5 $\mu \mathrm{m}$ particles was about 2 times higher than before cleaning.

Fig. 5 shows the size distribution of dust suspended in air in the process of vacuum cleaning. The data shown in Fig. 5(a) was obtained on a cloudy day with about $6 \mathrm{~mm}$ of rainfall, and the data shown in Fig. 5(b) was measured on a clear day that was after four weeks from the moving day. As shown in Fig. 5(a), dusts larger than $2 \mu \mathrm{m}$ in diameter were mainly suspended in the air on a rainy and cloudy day. Meanwhile, the dust larger than $1 \mu \mathrm{m}$ in diameter resuspended in air on a sunny day as shown in Fig. 5(b). When using a vacuum cleaner, the concentration of dust larger than $10 \mu \mathrm{m}$ in diameter was not high in comparison with the condition that a broom was used to clean a dusty floor on the moving day. 
(a)

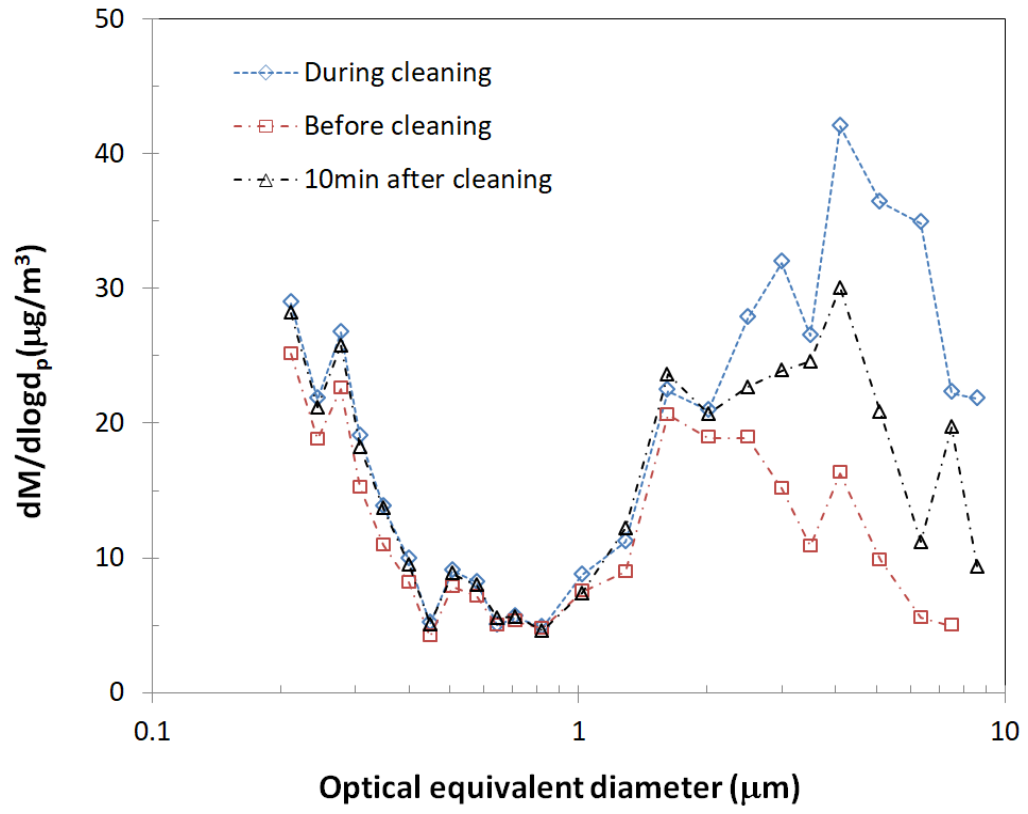

(b)

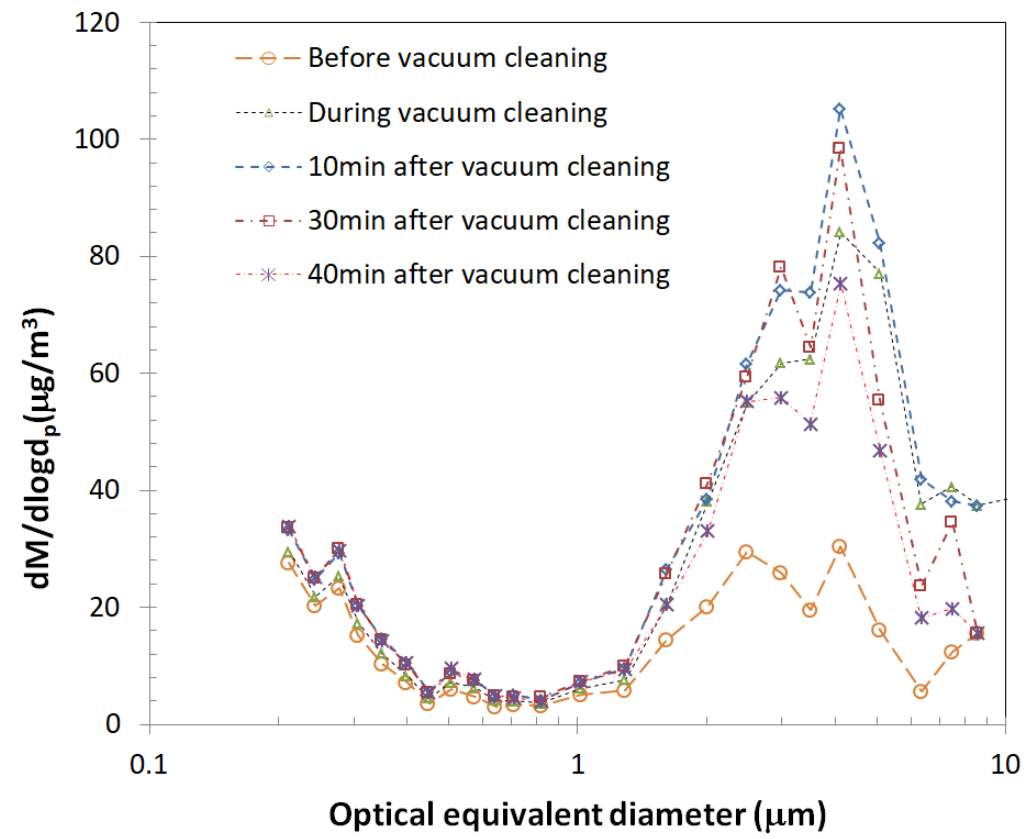

Fig. 5. Particle size distributions of resuspended dust during cleaning with a vacuum cleaner on a (a) rainy day and (b) sunny day.

Fig. 6 shows the changes in the suspended dust concentration before cleaning, during cleaning, and after cleaning with a vacuum cleaner on a clean day that is four weeks after the moving day. The $\mathrm{PM}_{10-2.5}, \mathrm{PM}_{2.5-1.0}$ and $\mathrm{PM}_{1.0}$ obtained by OPC are expressed as a ratio of mass concentrations, and the values measured by $\mathrm{CPC}$ are represented as the ratio $\left(\mathrm{N} / \mathrm{N}_{0}\right)$ of the number concentrations $(\mathrm{N})$. It was confirmed that dust larger than $1 \mu \mathrm{m}$ in diameter were suspended in the air. They floated for about 40 minutes despite the opening of the windows. The decrease in it was observed when natural ventilation was active. The number concentration measured by CPC increased rapidly at the beginning of the operation of the vacuum cleaner as shown in Fig. 6. As the production of particles in the vacuum cleaner sharply decreased, the particle concentration in the air decreased sharply.

Fig. 7 shows the change of the total number concentration variation of particles larger than $250 \mathrm{~nm}$ in diameter and $4 \mathrm{~nm}$ in diameter. They are measured by OPC and CPC, respectively. Most particles are smaller than $250 \mathrm{~nm}$ in diameter. When the sampling probe of the CPC is placed near to the air outlet of the vacuum cleaner, the higher number concentration of dusts was measured. This vacuum cleaner was not equipped with a HEPA filter at the outlet. The generation of highly concentrated particles shows a possibility that evaporation, nucleation, and condensation were made from the adhered organic substances on the 


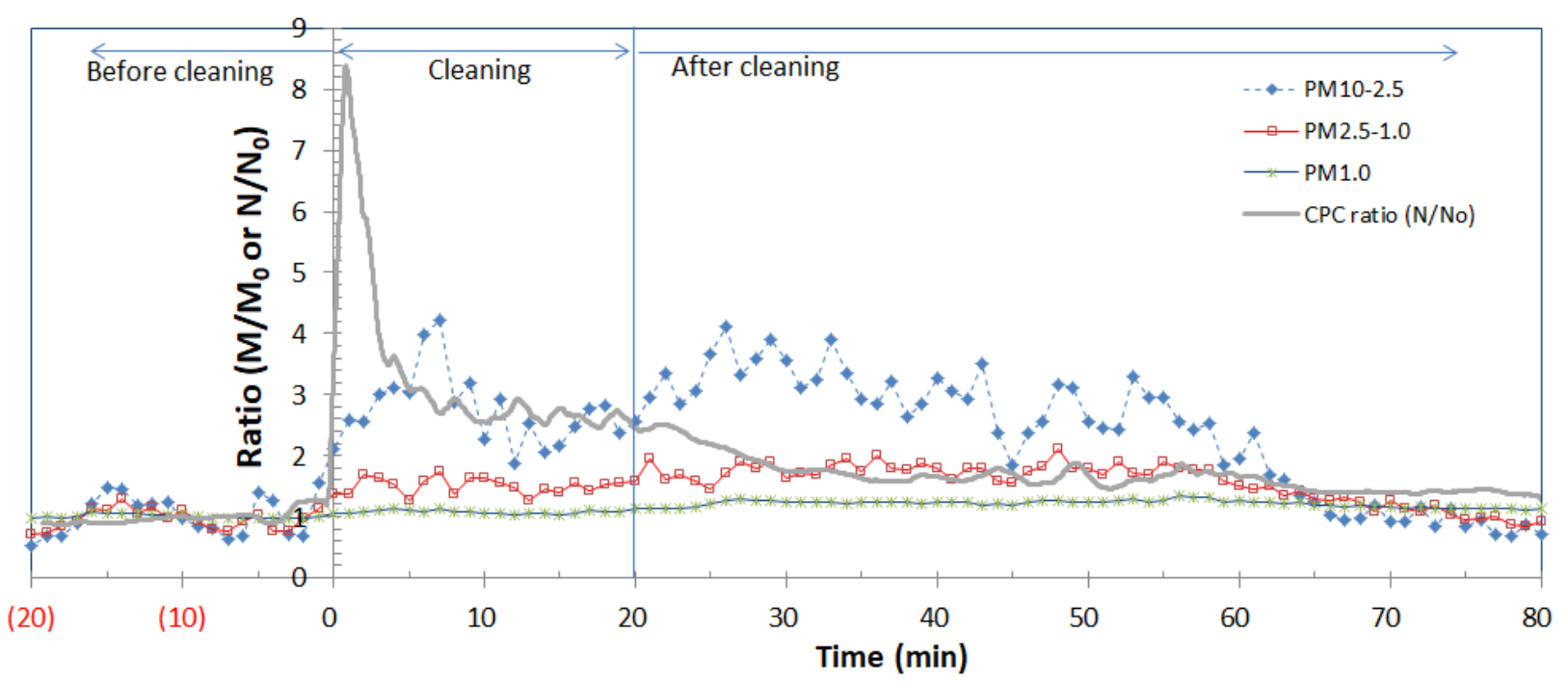

Fig. 6. Mass or number concentration ratios with time of particles during cleaning with a vacuum cleaner.

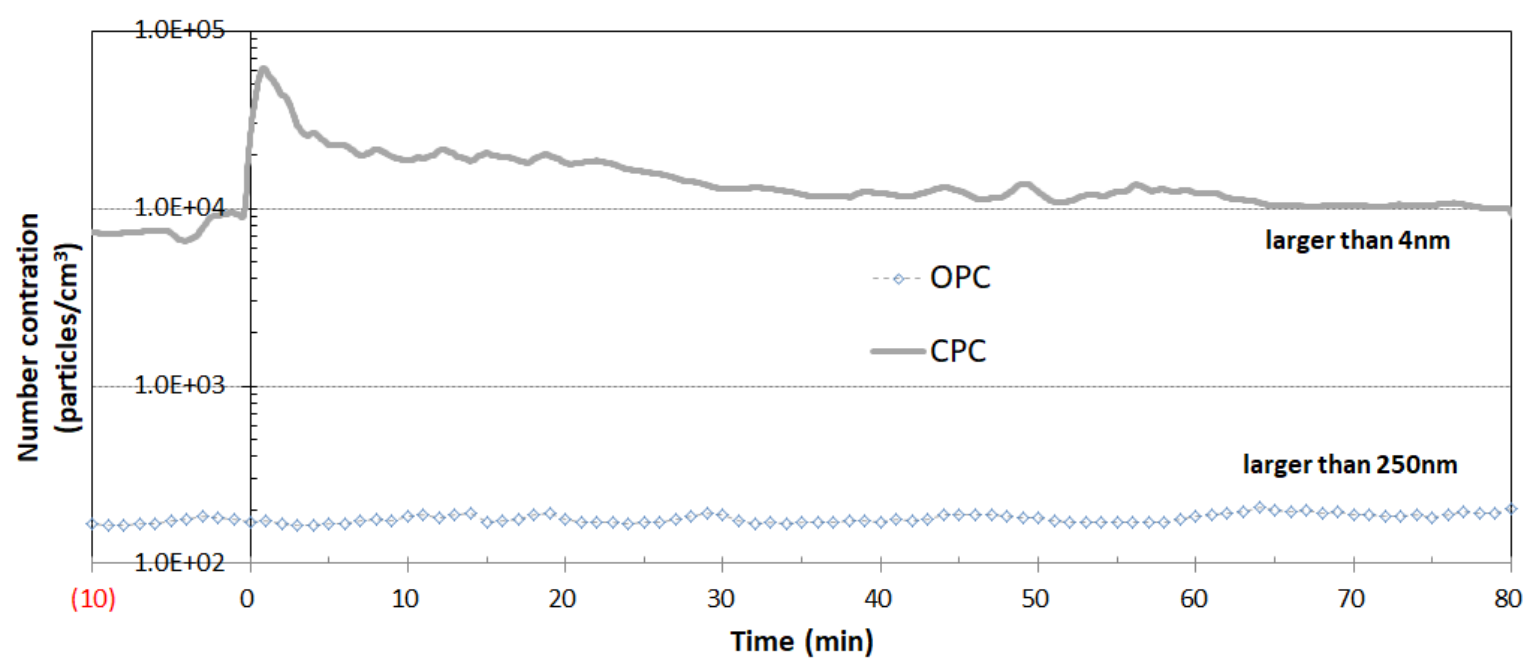

Fig. 7. Particle number concentrations with time during cleaning with a vacuum cleaner.

motor surface of vacuum cleaner. It is assumed that the heat generated by the motor of the vacuum cleaner has caused the nanoparticle formation. Further research is needed in the future.

\section{SUMMARY}

In this study, we investigated the characteristics of dust generated while cleaning an office where people wear shoes. The mass concentrations of the $\mathrm{PM}_{10}, \mathrm{PM}_{2.5}$, and $\mathrm{PM}_{1.0}$ and the size distributions of dust particles larger than $0.3 \mu \mathrm{m}$ in diameter were measured and analyzed during the cleaning. Specifically, the resuspension characteristics of fine floor dust during the operation of a broom and a vacuum cleaner were analyzed. The concentrations of the $\mathrm{PM}_{2.5}$ and $\mathrm{PM}_{10}$ increased when the cleaning began, and these particles floated indoors for a long time after the cleaning was finished. On the other hand, the $\mathrm{PM}_{1.0}$ concentration did not show a tendency to increase during the cleaning. In particular, while sweeping with a broom, the concentration of 1-2.5 $\mu \mathrm{m}$ dust particles increased by $\sim 5$ times, whereas that of $2.5-10 \mu \mathrm{m}$ particles increased by $\sim 60$ times when the floor was excessively dusty. The concentration of dust in the range of $2.5-10 \mu \mathrm{m}$ in diameter increased while vacuuming but did not exceed five times of the initial concentration. While operating the vacuum cleaner, which was equipped with an exhaust filter, the CPC-measured total particle number concentration rapidly increased during the initial stage of vacuuming and then decreased. Most of the particles produced while vacuuming were smaller than $250 \mathrm{~nm}$ in diameter.

In future work, we will systematically measure, analyze and characterize suspended particulates produced by indoor activities.

\section{ACKNOWLEDGMENTS}

This research was supported by the National Strategic Project-Fine Particle of the National Research Foundation of 
Korea (NRF) funded by the Ministry of Science and ICT (MSIT), the Ministry of Environment (ME), and the Ministry of Health and Welfare (MOHW) (2017M3D8A1091924).

\section{REFERENCES}

Bae, G.N. and Kim, J.B. (2017). Research trend on behavior and control of indoor aerosols. J. Odor Indoor Environ. 16: 99-112 (in Korean with English Abstract).

Knibbs, L.D., Congrong, H., Duchaine, C. and Morawska, L. (2012). Vacuum cleaner emissions as a source of indoor exposure to airborne particles and bacteria. Environ. Sci. Technol. 46: 534-542.

Lee, S.W., Heo, Y.M., Han, S.Y. and Kim, D. (2014). Emission characteristics of airborne particles and bacteria from household vacuum cleaners. J. Odor Indoor Environ. 13: 256-262 (in Korean with English Abstract).

Ozturk, B. (2006). A comparison of the performances of dry and wet vacuum cleaners for the control of indoor particulate matters. J. Int. Environ. Appl. Sci. 1: 107-119. Wu, C.L. Chao, C.Y.H., Sze-To, G.N., Wan, M.P. and Chan, T.C. (2012). Ultrafine particle emission from cigarette smoldering, incense burning, vacuum cleaner motor operation and cooking. Indoor Built Environ. 21: 782796.

Yoon, H, Shuai, J.F., Kim, T., Seo, J., Jung, D., Ryu, H. and Yang, W. (2017). Microenvironmental time-activity patterns of weekday and weekend on Korean adults. $J$. Odor Indoor Environ. 16: 182-186 (in Korean with English Abstract).

Received for review, October 14, 2019

Revised, October 14, 2019

Accepted, November 6, 2019 